\title{
Design and Fabrication of High Frequency Linear Function Generator with Digital Frequency Counter using MAX038 and a PIC microcontroller
}

\author{
P. KanakaRaju ${ }^{1}$ and M. PurnaChandra Rao ${ }^{2}$
}

Abstract - The main process of this research is to design and fabricate an extremely low cost, small sized, accurate and versatile function generator up to $17 \mathrm{MHz}$ with frequency resolution of $1 \mathrm{~Hz}$ by using minimum components like IC MAX038- analog function generator along with digital frequency counter implemented by PIC16F876 microcontroller and $2 \times 16$ characters LCD. External amplitude/duty cycle is controlled by ICs LT1364[1] and NE5532N[2], with the following key features: $1 \mathrm{~Hz}$ to $17 \mathrm{MHz}$ operating frequency range, sine, square and triangular waveforms, independent frequency and duty-cycle adjustments, $15 \%$ to $85 \%$ variable duty cycle along with $50 \%$ fixed duty cycle, low-impedance output buffer, low temperature drift. In this research, the complete design of the function generator is provided. Hardware and software technologies are integrated to fabricate this instrument.

Key words: function generator, frequency counter, PIC microcontroller, various waveforms.

Introduction: function generator is a versatile instrument, extensively used in electronics, mechanics, bioengineering, physics and many other fields. It generates a wide variety of electrical signals and waveforms for testing and diagnostic applications. Figure 1 shows the most common waveforms generated by a function generator. Many of the comprehensive function generators are able to operate at much higher frequencies: $1 \mathrm{~Hz}$ to $20 \mathrm{MHz}$.

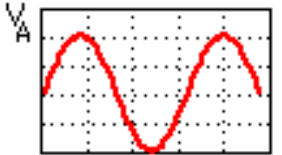

$\mathrm{t}(\mathrm{s})$

SINE

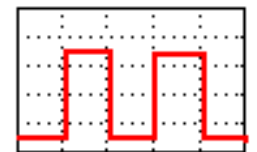

t (s)

SQUARE

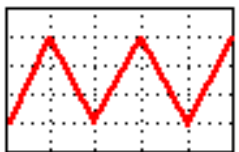

t (s)

TRIANGULAR

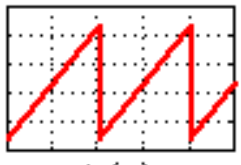

$\mathrm{t}$ (s)

RAMP

Figure 1: Waveforms generated by a function generator

There are many ways of designing function generator circuits, however there are two main approaches:

- Analogue function generator: It utilize analogue technology and offer a number of advantages:

1. Cost effective: Analog function generators are very cost effective, being at the lower end of the function generator price range.

2. Simple to use: Analog function generators provide an effective test instrument that is able to meet most user needs.

\footnotetext{
${ }^{1}$ Department of Electronics and Physics, GIS, GITAM University, Visakhapatnam, INDIA

${ }^{2}$ Department of System Design, Andhra University, Visakhapatnam, INDIA
} 
3. Maximum frequencies: The analog function generators do not have the high frequency limitations on non-sinusoidal waveforms such as triangles and ramps as do the digital function generator.

- Digital function generator: It utilizes digital technology to generate the waveforms, such as Direct Digital Synthesis [6]. DDS uses a phase accumulator, a look-up table containing a digital representation of the waveform, and a DAC [8]. These are able to offer high levels of accuracy and stability, but the disadvantage is that they are more comprehensive than analogue cousins; they require a high performance $\mathrm{DAC}$ and other digital circuitry which are costly and also complicated.

This research work mainly deals with the design and fabrication of analog function generator with IC MAX038.

\section{Description:}

MAX038: IC MAX038 is a high-frequency, precision function generator producing highfrequency triangle, sawtooth, sine, square, and pulse waveforms with a minimum of external components. The output frequency can be controlled over a frequency range of $0.1 \mathrm{~Hz}$ to $20 \mathrm{MHz}$ [3] by an internal $2.5 \mathrm{~V}$ band gap voltage reference and an external resistor and a capacitor. The duty cycle can be varied over a wide range by applying a $\pm 2.3 \mathrm{~V}$ control signal, facilitating pulse-width modulation and the generation of sawtooth waveforms. Frequency modulation and frequency sweeping can also be achieved. The duty cycle and frequency controls are independent. Sine, square, or triangle waveforms can be selected at the output by setting the appropriate code at two TTL-compatible select pins. The output signal for all waveforms is a $2 \mathrm{~V}_{\mathrm{P}-\mathrm{P}}$ signal that is symmetrical around ground. The low-impedance output can drive up to $\pm 20 \mathrm{~mA}$. The TTL-compatible SYNC output from the internal oscillator maintains a $50 \%$ duty cycle regardless of the duty cycle of the other waveforms to synchronize other devices in the system.

PIC16F876: The PIC16F876 is a high performance RISC CPU [4] with 35 single word instructions, CMOS FLASH-based 8-bit microcontroller packs Microchip's powerful PIC® architecture in 28-pin package. Features: 256 bytes of EEPROM data memory, self programming, an ICD, 5 channels of 10-bit Analog-to-Digital (A/D) converter, 2 additional timers, 2 capture/compare/PWM functions, the synchronous serial port can be configured as either 3-wire Serial Peripheral Interface (SPI $\left.{ }^{\mathrm{TM}}\right)$ or the 2-wire Inter-Integrated Circuit $\left(\mathrm{I}^{2} \mathrm{C}^{\mathrm{TM}}\right)$ bus and a Universal Asynchronous Receiver Transmitter (USART). All of these features make it ideal for more advanced level A/D applications in automotive, industrial, appliances and consumer applications.

Figure 2 shows the basic circuit for the generation of sine wave as output with $50 \%$ duty cycle[3]. 


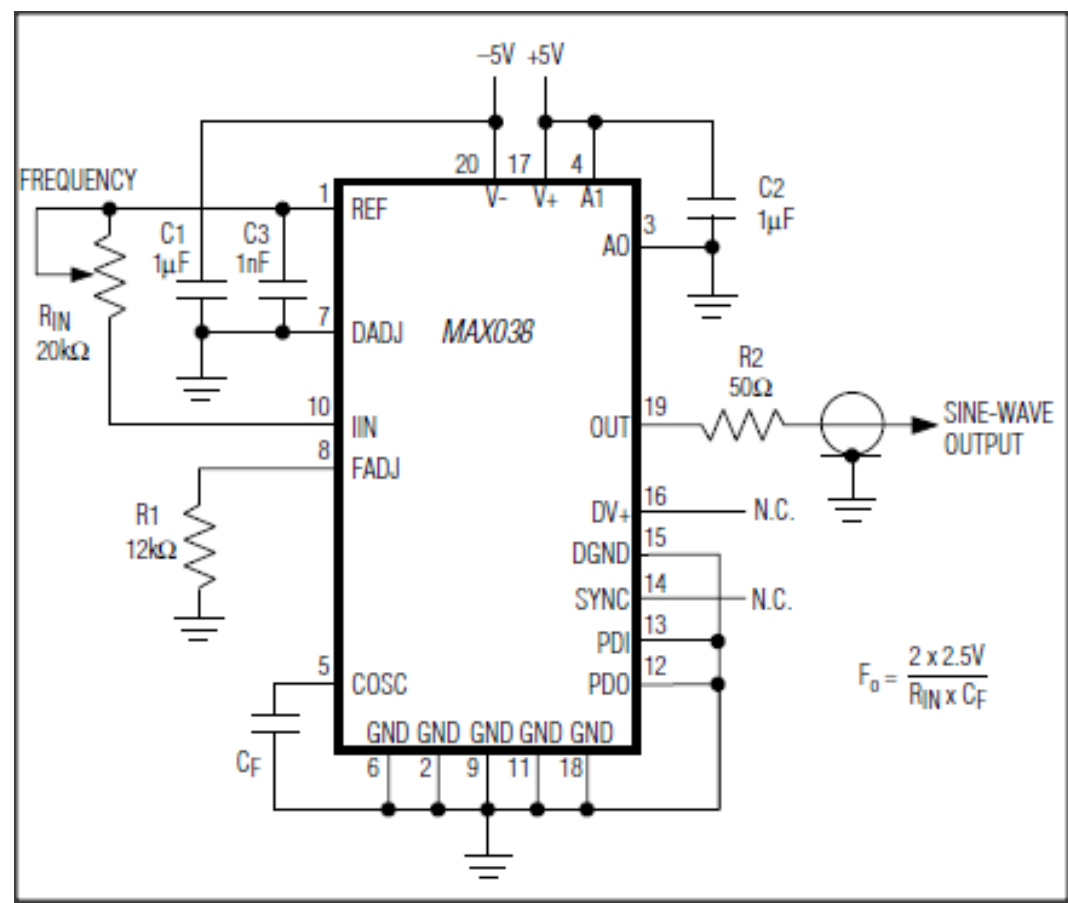

\section{2c. Basic circuit of sine wave output with $50 \%$ duty cycle}

Design and Fabrication: $\quad$ MAX038 is a relaxation oscillator, operates by alternately charging and discharging a capacitor, with constant currents. Basically it is a dual slope integrator that simultaneously produces a triangular and a square wave (TTL). The frequency is determined by the external oscillator capacitor and the current flowing into IIN. This triangular wave is applied to an internal comparator, in order to make a square wave. By applying the triangular wave to a waveform shaping circuit, it produces a sine wave with constant amplitude[5]. The triangle, square, and sine waves are input to a multiplexer that selects the type of wave which is applied to the low impedance separating amplifier [7]. Figure 3 and 4 shows the block diagram and the circuit diagram of the function generator respectively.

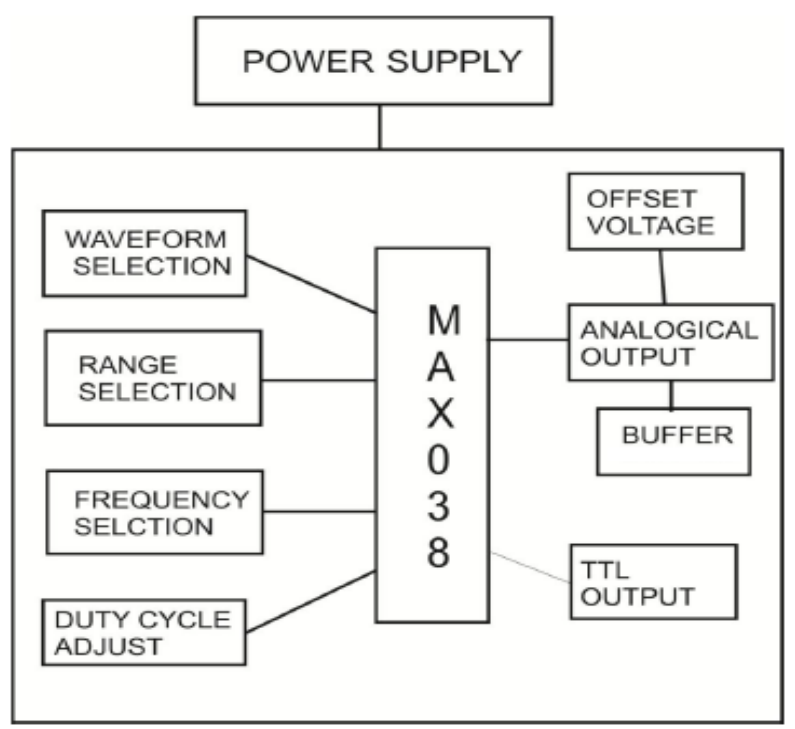

Figure: 3 Block diagram of the function generator 

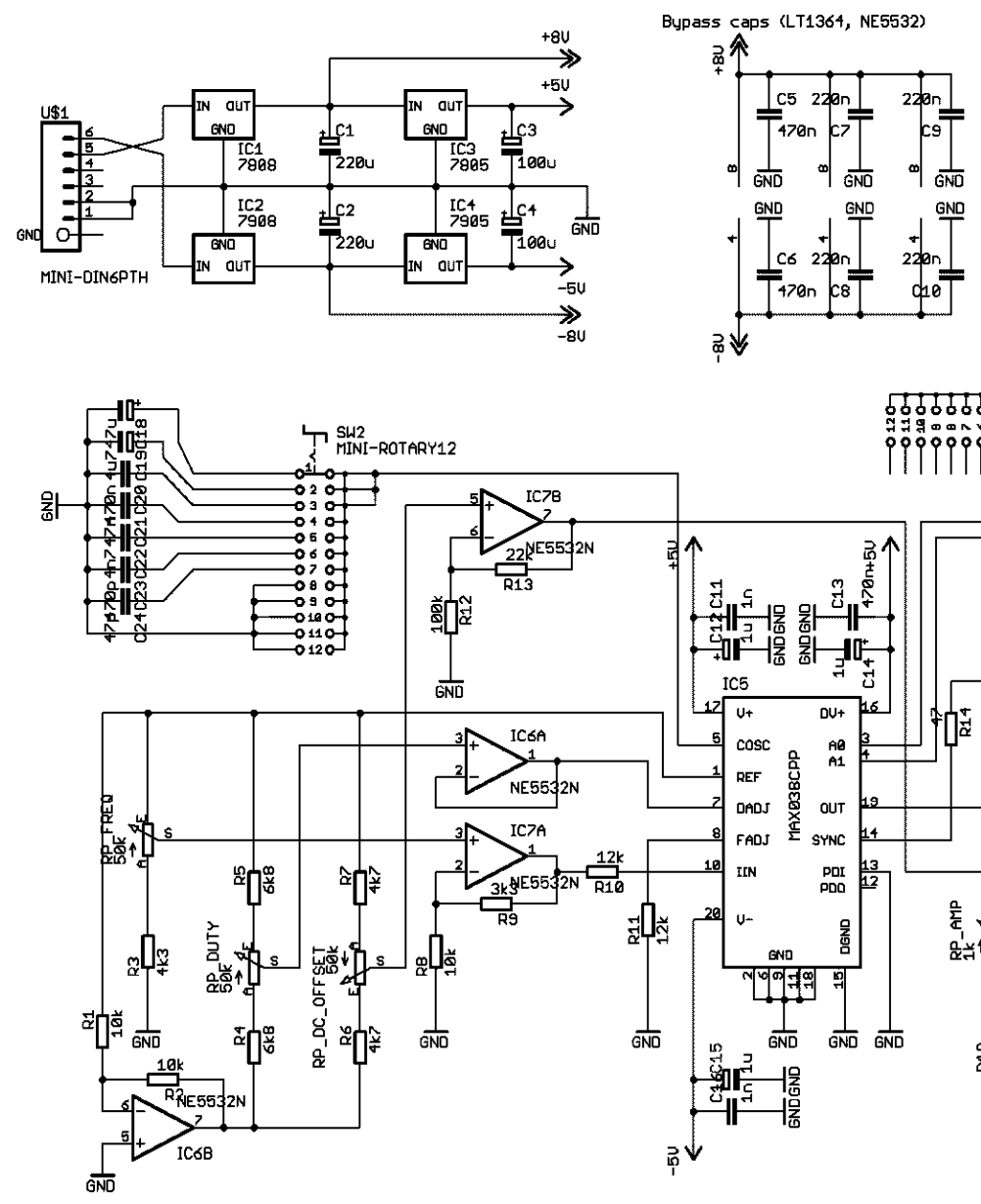

Figure: 4 Circuit diagram of the function generator
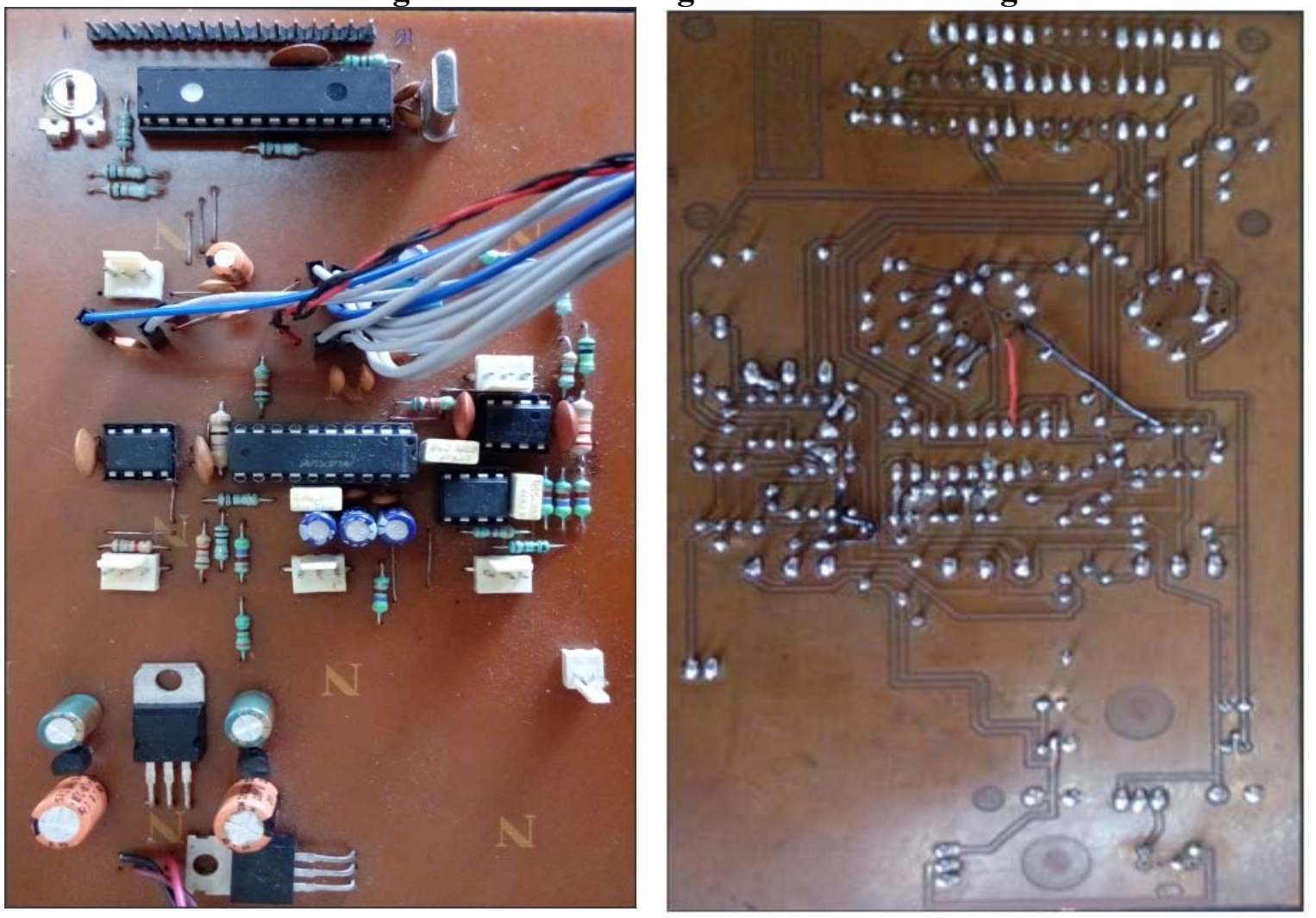


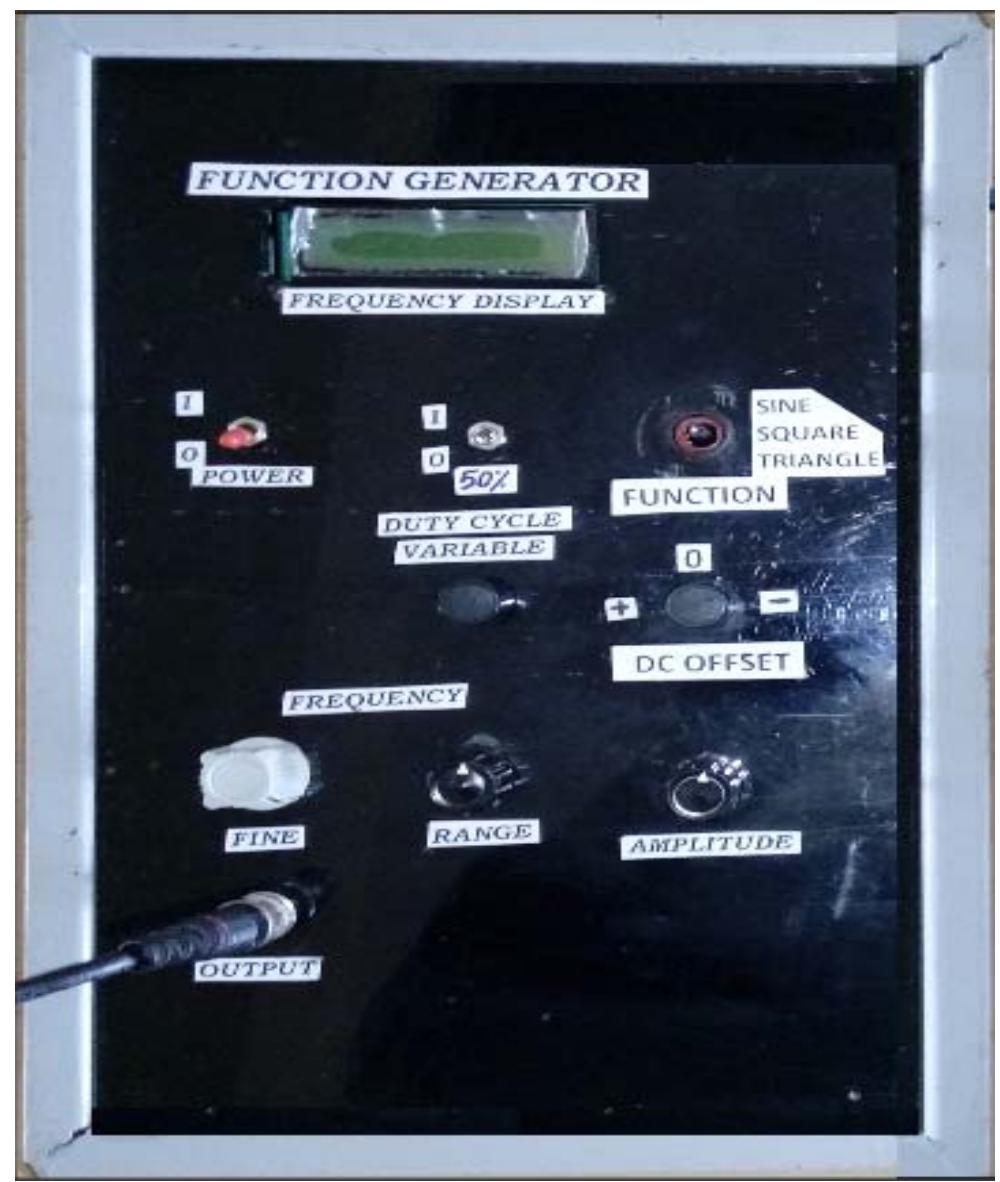

Figure: 6 complete function generator with front panel controls

As shown in figure 6, the frequency range can be selected by placing different capacitors to the COSC input of MAX038 by using a twelve position rotary switch. The output frequency can be selected within the frequency range by a potentiometer [8] for the fine tuning. The DADJ input of MAX038 controls the duty cycle of the waveform. By using a switch, it is possible to either enable or disable the $50 \%$ fixed duty cycle. If enabled, the duty cycle can be adjusted by a potentiometer. The type of output waveform can be selected from a "FUNCTION" switch which is a 3-position rotary switch. The common of the switch is driven HIGH, while the two inputs of MAX038- A0 and A1 are pulled LOW with $10 \mathrm{~K}$ resistors. When both inputs $\mathrm{A} 0$ and $\mathrm{A} 1$ are ' 0 ': the output is square wave, when $\mathrm{A} 0$ is ' 1 ' and A1 is ' 0 ': the output is triangular waveform and when A1 is ' 1 ' and A0 is ' 0 ': the output is sine wave. The output is buffered with LT1364 operational amplifier that can linearly amplify the waveform up to $5 \mathrm{Vpp}$. The peak to peak amplitude is also controlled a potentiometer. By default, the output waveform is symmetrical to the ground. DC offset can be added to the output by a switch "DC OFFSET ". The DC offset is also controlled by a potentiometer. Finally the output is connected to a BNC connector for external use.

Results and discussion: After the completion of design and fabrication of the function generator, its performance was tested in terms of nature of the waveform, its amplitude and the time interval by a $\mathrm{CRO}$, which are at a good level of satisfaction. The type of the function (sine, square and triangle) which appears on the CRO screen exactly matches with what LCD shows. The frequency shown by the function generator's LCD is tested by a CIE122 model multimeter, as well as with a digital CRO, the frequency value on the LCD exactly matches with the multimeter value as well as with digital CRO value. Below figures $7 \mathrm{a}, 7 \mathrm{~b}, 7 \mathrm{c}, 7 \mathrm{~d}, 7 \mathrm{e}$ and $7 \mathrm{f}$ are evidence for the function generator's performance. In 
the figure, $\mathrm{CRO}$ shows the type of the waveform selected and multimeter shows the frequency in $\mathrm{KHz}$ that matches with function generator's LCD display in terms of $\mathrm{Hz}$.

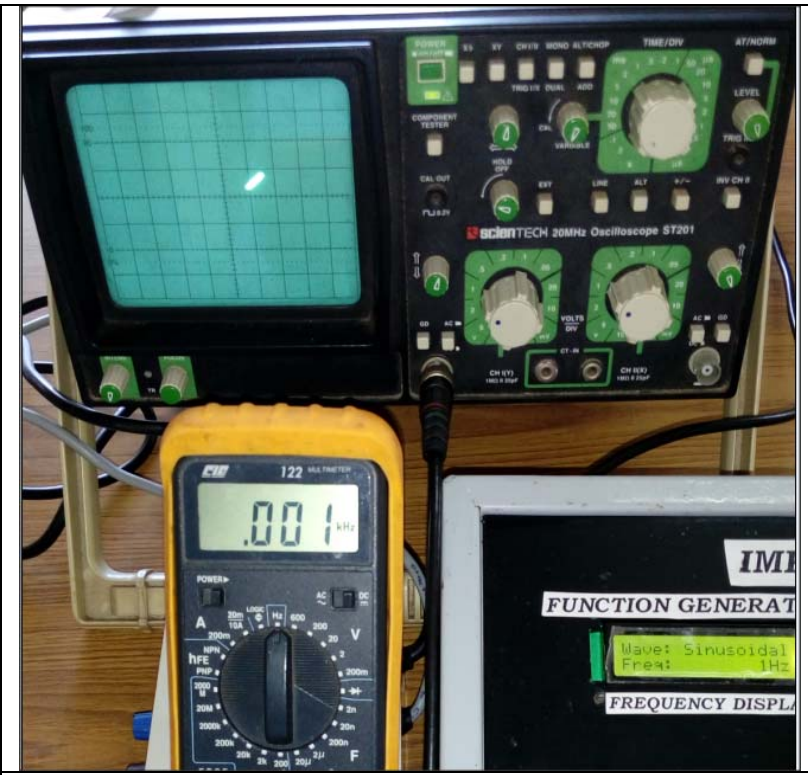

7a. Function generator at a frequency of $1 \mathrm{hz}$ (sinusoidally oscillating dot on the CRO screen)

Multimeter shows $0.001 \mathrm{KHz}$, LCD shows $1 \mathrm{~Hz}$

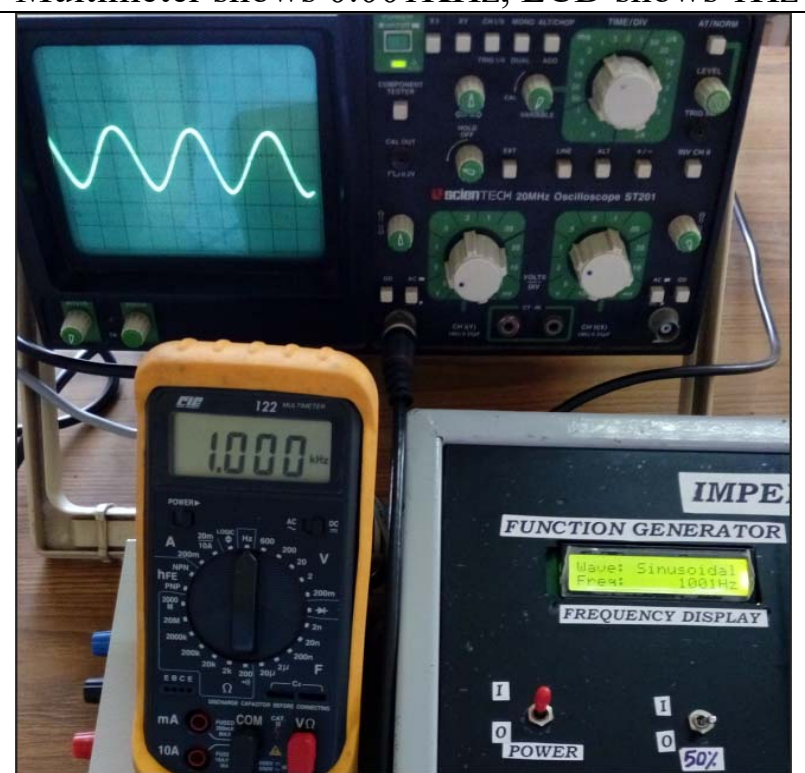

7c. Function generator at a frequency of 1Khz (Function: sinusoidal wave on the CRO screen)

Multimeter shows $1.000 \mathrm{KHz}, \mathrm{LCD}$ shows $1000 \mathrm{~Hz}$

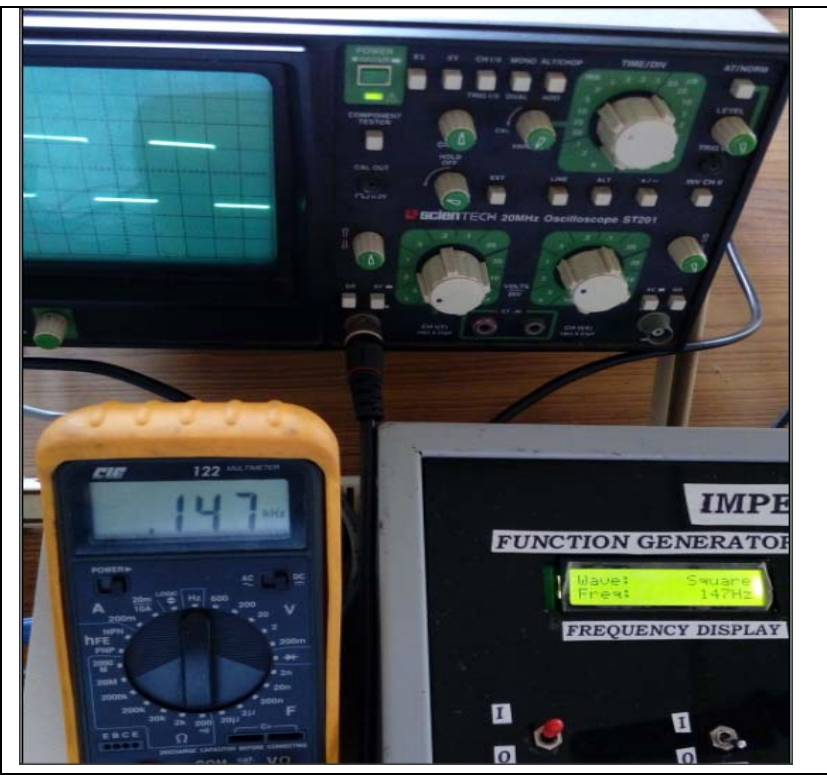

7b. Function generator at a frequency of 147 hz (Function: square wave on the CRO screen) Multimeter shows $0.147 \mathrm{KHz}$, LCD shows $147 \mathrm{H}$

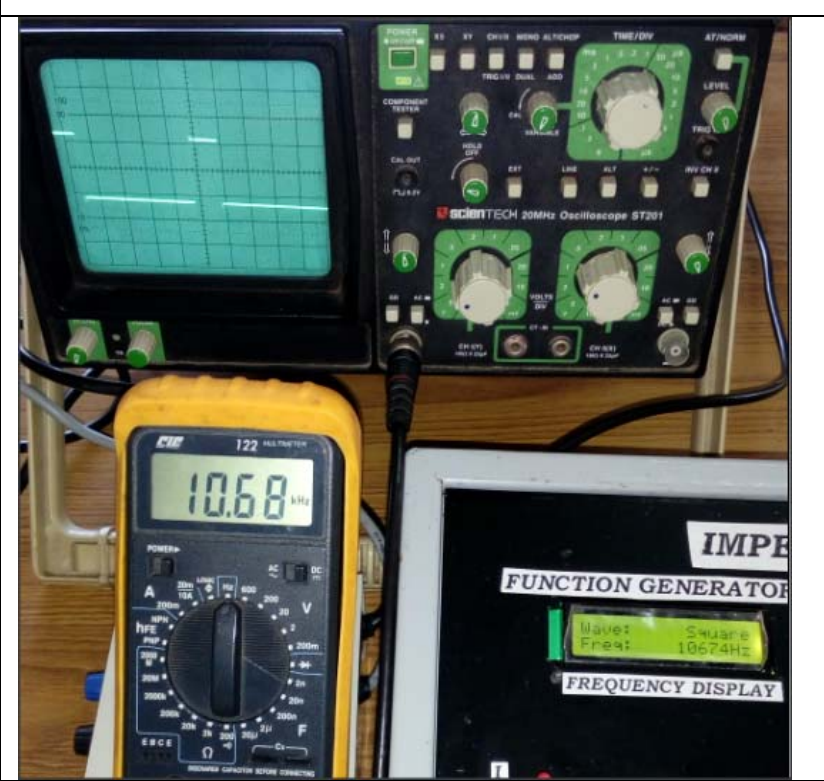

7d. Function generator at a frequency of $10.68 \mathrm{Khz}$

(Function: square wave with variable duty cycle on the CRO screen)

Multimeter shows $10.68 \mathrm{KHz}$, LCD shows $10674 \mathrm{~Hz}$ 


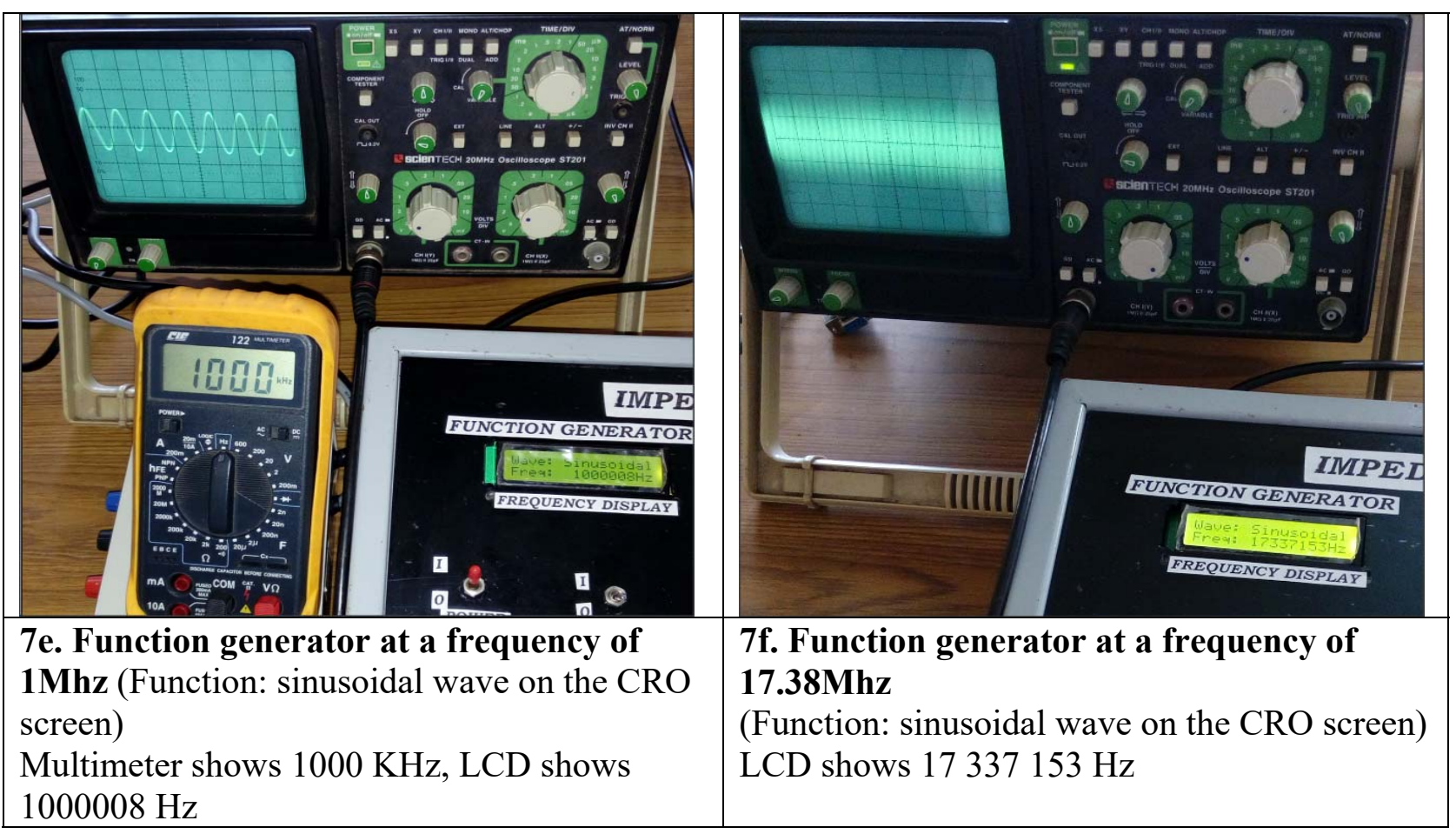

Below graph shows a plot of obtained frequency $\{\log ($ of $)\}$ compared with the standard frequency $\{\log$ (sf) $\}$ at 15 events, both are tracking each other. Thus the designed function generator working with almost nil error.

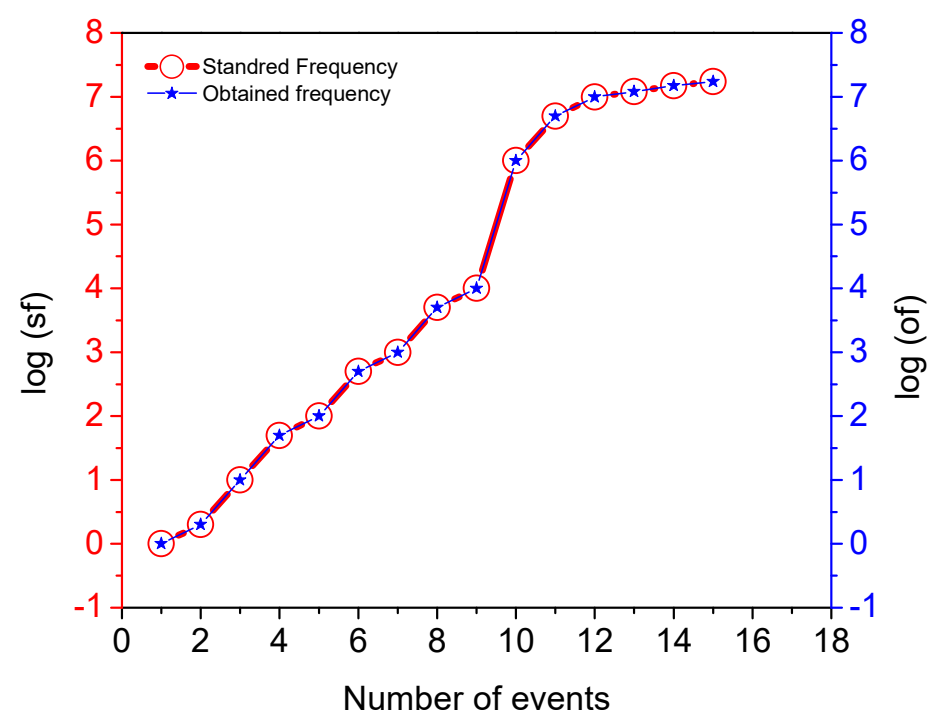

Graph: Obtained frequency $\{\log ($ of $)\}$ compared with the standard frequency $\{\log ($ sf $)\}$ at 15 events 
Conclusion: This paper describes the design and fabrication of low cost function generator useful for research and educational purpose. It is very easy to operate between the desired frequency and amplitude. The front panel controls are user friendly. Experimental results showed that the signal generator is of high precision, small size, and convenient and stable in use However, when the output frequency increases to $10 \mathrm{MHz}$, the amplitude begins to decay due to bandwidth limitations of the ic LT1364. Further improvements are needed.

\section{References}

[1] http://cds.linear.com/docs/en/datasheet/13645fa.pdf

[2] https://www.fairchildsemi.com/datasheets/NE/NE5532.pdf

[3] http://pdfserv.maximintegrated.com/en/ds/MAX038.pdf

[4] http://microrato.ua.pt/main/Actividades/Estagios/docs/pic16f87x.pdf

[5] C. F. Coombs, "Electronic Instrument Handbook," McGraw-Hill, Inc., New York, 1995.

[6] Jian Qi,1 Qun Sun, ${ }^{1}$ Xiaoliang Wu, ${ }^{2}$ Chong Wang, ${ }^{1}$ and Linlin Chen ${ }^{1}$, "Design and Analysis of a Low Cost Wave Generator Based on Direct Digital Synthesis".

[7] Xian Qin Han, Xiang Lei Zhu, "Multi-Signal Generator Design".

[8] Patrick O. Olabisi ${ }^{1}$, B. J. Olufeagba ${ }^{2}$ " "Step-Wise Approximation Technique in the Design of a Function Generator".

[9] Y. Sun, J. Lu, S. Liu, and H. Ben, "Design of sinusoidal signal generator based on AD9833 and potentiometer," Electrical Measurement \& Instrumentation, vol. 7, pp. 93- 96,2012. 